\title{
Rat model of heterotopic heart transplantation to investigate relevant donor heart harvesting method
}

\author{
Mukhammad Kayumov ${ }^{1}$, Hwa-Jin Cho ${ }^{2}$, Do-Wan Kim ${ }^{3}$, Kyo-Seon Lee ${ }^{3}$, In-Seok Jeong ${ }^{3}$ \\ ${ }^{1}$ Department of Thoracic and Cardiovascular Surgery, Chonnam National University Medical School, Gwangju, Korea \\ ${ }^{2}$ Division of Cardiology, Department of Pediatrics, Chonnam National University Hospital, Gwangju, Korea \\ ${ }^{3}$ Department of Thoracic and Cardiovascular Surgery, Chonnam National University Hospital, Gwangju, Korea
}

Background: Various preservation methods have been describing to elongate implantable time of the donor heart as well as to eliminate post-transplant graft failure. There have been increasing concern about hypothermic preservation technique that most of the researchers associate with increased mortality. Present study evaluates the efficacy of both hypothermic and sub-normothermic harvesting techniques on murine models.

Methods: The hearts were extracted from the donor rats (n-5/group) either after cardioplegic (CPS) infusion (HTK, $50 \mathrm{~mL}$ at $\left.24^{\circ} \mathrm{C}-26^{\circ} \mathrm{C}\right)$ or topic ice slush application $\left(0^{\circ} \mathrm{C}-4^{\circ} \mathrm{C}\right)$ without cardioplegic perfusion and heterotopically transplanted into the abdomen of the syngeneic recipients. The total donor heart ischemic time and heart beating initiation time were recorded intraoperatively. Graft function was examined by daily palpation and hearts were taken to histologic examinations after 2 weeks of survival.

Results: Nine of ten rats survived until 2 weeks without acute or chronic rejection. One rat excluded in the cardioplegia group which died in 10 days of survival after a sudden initiation of systemic inflammation. The mean ischemic time of the donor heart was 42.5 minutes in the cardioplegic group and 38 minutes in the hypothermic group. The heart generally started to beat within the 5 second of reperfusion with normal rhythm in the cardioplegic group rats whereas 10 minutes of reperfusion was needed in the hypothermic group until graft reaches to the normal rhythm. All hypothermic group hearts experienced fibrillation and irregular rhythms within the 5 minutes of reperfusion and slight massage support was needed in all hypothermic rats to initiate contraction.

Conclusions: Subnormothermic CPS perfusion showed comparable results in early outcomes. We will compare the result between normothermic and hypothermic CPS perfusion methods with laboratory and histologic examinations in the next trials.

Corresponding author: In-Seok Jeong

E-mail: isjeong1201@gmail.com

(c) The Korean Society for Transplantation

This is an Open Access article distributed under the terms of the Creative Commons Attribution Non-Commercial License (http://creativecommons.org/licenses/by-nc/4.0/) which permits unrestricted non-commercial use, distribution, and reproduction in any medium, provided the original work is properly cited. 